\title{
Laparoscopic Resection of An Extensive Tubo-ovarian Abscess in A Mechanical Valve Replacement Woman
}

\author{
Phornsawan WASINGHON ${ }^{1,2}$, Kuan-Gen HUANG ${ }^{2,3}$ \\ Phitsanulok, Thailand

\begin{abstract}
The case is a 44-year-old-female who presented with right lower abdominal pain without fever or leukocytosis. She had previous mechanical valve replacement with taking warfarin for last ten months from the diagnoses of infective endocarditis. The ultrasonography showed bilateral complex ovarian mass. At laparoscopy, the patient had a right tubo-ovarian abscess and a left mature teratoma with extensive adhesions to the rectum. A case illustrates an uncommon premenopausal age with right tuboovarian abscess and left mature teratoma. The laparoscopic surgery is an excellent benefit for quicker recovery time, smaller incisions, less post-operative pain, and especially of a few infections that will affect to underlying disease of the patient.
\end{abstract}

Keywords: Laparoscopy, Mechanical valve replacement, Ovarian mass, Tubo-ovarian abscess

Gynecol Obstet Reprod Med 2020;26(3):232-234

\section{Introduction}

Pelvic inflammatory disease (PID) is a prevalent medical problem among reproductive age and menopausal women. One particular pelvic inflammatory disease, which is a severe sequela, is a tuboovarian abscess (TOA). In addition, it has been reported that $34 \%$ of patients who were initially diagnosed with PID had an occurrence of tuboovarian abscess (1-3) in which the tuboovarian abscess patients were asymptomatic surgically confirmed with $35 \%$ of patients being afebrile, and $23 \%$ had an average white blood cell (WBC)

${ }^{1}$ Department of Obstetrics and Gynecology, Buddhachinnaraj Hospital and Naresuan University, Phitsanulok, Thailand.

2 Department of Obstetrics and Gynecology, Chang Gung Memorial Hospital at Linkou, Taoyuan, Taiwan.

${ }^{3}$ Chang Gung University, College of Medicine, Kweishan, Taoyuan, Taiwan.

\begin{tabular}{|c|c|}
\hline Address of Correspondence: & $\begin{array}{l}\text { Kuan-Gen Huang } \\
\text { Department of Obstetrics and } \\
\text { Gynecology, Chang Gung Memorial } \\
\text { Hospital, Linkou Medical Center and } \\
\text { Chang Gung University College of } \\
\text { Medicine. 5, Fuxing Street, Kweishan, } \\
\text { Taoyuan, Taiwan } 333 \\
\text { kghuang@ms57.hinet.net }\end{array}$ \\
\hline $\begin{array}{l}\text { Submitted for Publication: } \\
\text { Accepted for Publication: }\end{array}$ & $\begin{array}{l}16.11 .2018 \\
05.01 .2019\end{array}$ \\
\hline ORCID IDs of the authors: & $\begin{array}{l}\text { PW: 0000-0001-9530-9438. } \\
\text { KGH: 0000-0001-8481-8077 }\end{array}$ \\
\hline \multirow{3}{*}{ 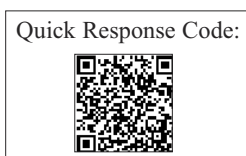 } & Access this article online \\
\hline & $\begin{array}{l}\text { Website: www.gorm.com.tr } \\
\text { e- mail: info@gorm.com.tr }\end{array}$ \\
\hline & DOI:10.21613/GORM.2018.858 \\
\hline
\end{tabular}

How to cite this article: Wasinghon P. and Huang KG. Laparoscopic Resection of An Extensive Tubo-ovarian Abscess in A Mechanical Valve Replacement Woman. Gynecol Obstet Reprod Med. 2020;26(3):232-234 count (2). The symptoms of PID include adnexal tenderness, cervical excitation, pyrexia, vaginal discharge, an elevated white cell count, elevated erythrocyte sedimentation rate (ESR), elevated C-reactive protein, positive test of Neisseria Gonorrhea or Chlamydia Trachomatis, and adnexal mass by imaging. A laparoscopy or laparotomy with drainage of the abscess, unilateral or bilateral salpingo-oophorectomycor pelvic clearance are generally the surgical approaches for a tuboovarian abscess. If women are suitable for a laparoscopy and the surgeon has experienced laparoscopic skills, then this generates benefits for quicker recovery time, smaller incisions, and less post-operative pain (3). This study presented the case of a patient with afebrile chronic pelvic pain and the bilateral ovarian masses, who had an underlying mechanical valve replacement and used warfarin. The final diagnosis was a right tuboovarian abscess and a left dermoid cyst with extensive adhesion.

\section{Case Report}

A 44-year-old-female, who had three cesarean deliveries, sustained underlying hypertension (HT). She had a chest wall scar from infective endocarditis where the mechanical valve had been replaced since August 2017 then she was prescribed warfarin. She had right lower abdominal pain for ten months, progressively. The body mass index was $22.1 \mathrm{~kg} / \mathrm{m}^{2}$; usual vital signs with no fever. The pelvic mass was about 14 weeks in size of the uterine reference site, which the ultrasonography showed the bilateral complex cystic-solid ovarian masses on the right and left, $6.5 \mathrm{~cm}$ and $7.1 \mathrm{~cm}$ in size, respectively. The right ovarian mass showed a thickened endosalpingeal fold. The left ovarian mass showed delicate echoic lines emerging in the dermoid content. The level of CA 125 was $56.3 \mathrm{U} / \mathrm{mL}$. The provisional diagnosis was a right tubo-ovarian abscess 
and a left mature teratoma. Owing to complete family with three children, the patient desire for definitive surgery to remove both adnexal masses and a uterus which she could not suffer from many time of the procedures. The patient had been counsel before the recommended treatment was a laparoscopy. The plan of management was a laparoscopic hysterectomy and bilateral salpingo-oophorectomy (BSO). After the underlying disease was stabilization with the cardiovascular medicine doctor, the preoperative warfarin intake was stopped five days before the operation and changed to a heparin infusion with an initial bolus dose of 80 units $/ \mathrm{kg}$ followed by the maintenance dose of 18 units/ $\mathrm{kg} /$ hour every six hours. The heparin was stopped six hours before the surgery. The problematic intraoperative surgery was lysis of adhesion at the obliterated cul-de-sac from the rectum, which was attached to both adnexae (Figure 1). The pus was composed of an intraoperative leakage from the right ovarian mass (Figure 2).

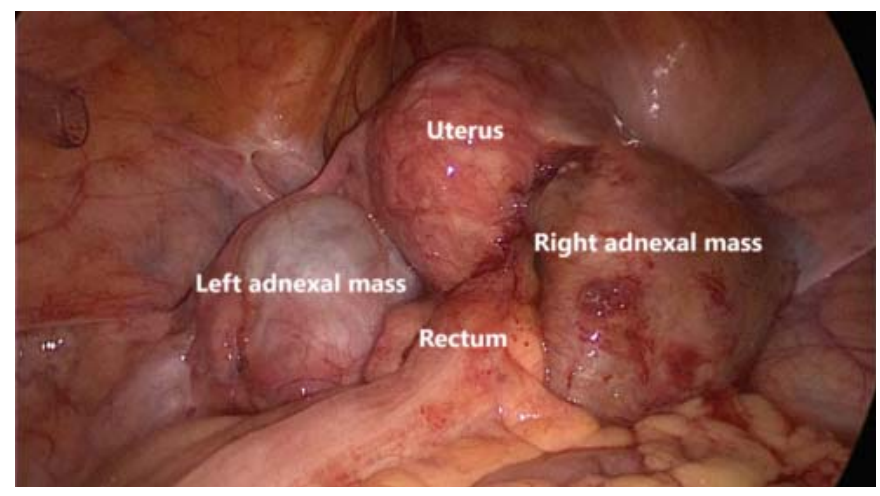

Figure 1: Laparoscopic view of the uterus and bilateral adnexal masses with obliterative cul-de-sac from extensive adhesions at the rectum

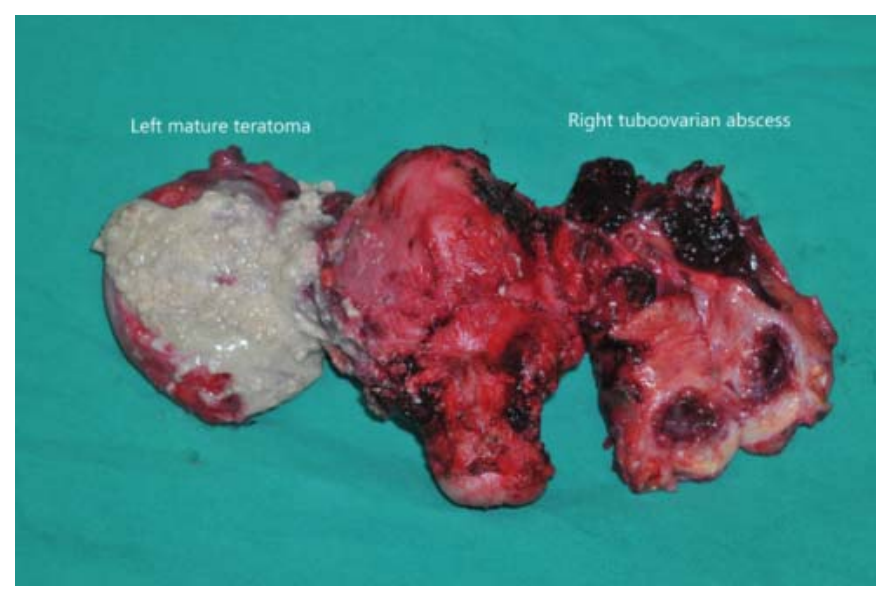

Figure 2: The gross specimen showed a right tubo-ovarian abscess, a left mature teratoma, and normal uterine cervix

The severe adhesion was lysis by the hydrodissection technique with the infiltration of sterile water between the serosal surface of the uterus, both adnexae, and the rectum. The uterus and both adnexae were steps for the removal of the laparoscope with minimal blood loss of $50 \mathrm{~mL}$. The heparin was restarted for infusion at 48 hours following the post-operative period. The patient was discharged on the seventh postoperative day with a target international normalized ratio (INR) range of 2 to 3 of warfarin. There were no postoperative complications. The histopathology showed the abscess formation at the right ovary and the mature teratoma at the left ovary. This case is the high risk for surgery in a patient who coexists with the high risk of infection from the tuboovarian abscess and the extensive adherent adhesion at Chang Gung Memorial Hospital. The study obtained the exemption requirement approval by the institutional review board (IRB). Written informed consent was obtained from the patient.

\section{Discussion}

A tuboovarian abscess patient had admitted at Chang Gung Memorial Hospital, Taiwan with chronic pelvic pain. This kind of pain is a possible long term complication in onethird of women with tuboovarian abscess and is related to the severity and number of episodes (4). The incidence of chronic pelvic pain has been shown to be $12 \%, 30 \%, 67 \%$ after one episode, two episodes, and three or more episodes of PID or tuboovarian abscess, respectively(5). Crespo et al. showed that the treatment with antibiotics were no statistical differences observed with those managed surgically regarding chronic pain (6). The imaging study revealed a bilateral ovarian mass in this patient. Ultrasonography is usually the first radiographic study of choice in evaluating a suspected abdominal mass or pelvic mass. The patient was afebrile and had a normal white blood cell count although she had complaints of abdominal pain with radiologic evidence of bilateral adnexal masses. The right ovarian mass showed a thickened endosalpingeal fold. Timor-Tritsch et al. believe that the endosalpingeal fold sign is pathognomonic of acute tubal inflammation and suggestive of a "cogwheel" sign (5). The incomplete septa within the tubes is a sensitive sign of tubal inflammation or an abscess (7). The left dermoid cyst or mature teratoma was disclosed by the ultrasonography of hyperechoic lines within the mass content. Mos reported the hyperechoic lines are produced by the presence of hair cells (8). Although the reported to drainage of the abscess with concomitant use of antibiotics has been adopted with the success rate of 80 to $100 \%$ following either ultrasound-guided or the computed tomography guided drainage (9). This patient had been diagnosed of a right tuboovarian abscess and a left dermoid cyst by the ultrasonography with chronic pelvic pain and afebrile illness. The drainage was not suitable for this patient due to the presenting symptom of the chronic pelvic pain, and a left dermoid cyst could not be improvement clinically. In view of the underlying disease, the disease was controlled with oral anticoagulant medication while the oral anticoagulants was administered after the valve's implantation to decrease the risk of thromboembolism for ten months. The bridging therapy with either intravenous unfractionated heparin or low-molecular-weight heparin evolved empirically to reduce the thromboembolic events during the temporary interruption of oral anticoagula- 
tion in a high-risk patient such as mechanical valve replacement (MVR). In general, the administration of anticoagulant medication is stopped three to four days before the procedure. When the oral anticoagulant was stopped, the bridging anticoagulation with intravenous unfractionated heparin or subcutaneous low-molecular-weight heparin is usually started before surgery 36 to 48 hours when the international normalized ratio (INR) falls below the therapeutic threshold (INR of 2.0 or 2.5). The anticoagulant is then stopped four to six hours for intravenous unfractionated heparin, or 12 hours for subcutaneous low-molecular-weight heparin before the procedure. Then, the anticoagulant is restarted postoperatively as soon as primarily 12 to 24 hours after surgery (10). Accordingly, decisions about bridging should be individualized and should account for the compromise between thrombosis. The patient of the present study's preoperative anticoagulation medication was stopped five days before the operation and changed to an intravenous unfractionated heparin infusion, which the heparin was stopped six hours before the surgery. The heparin was restarted for infusion at 48 hours following the post-operative period. The patient was discharged on the seventh postoperative day with a target INR range of 2 to 3 of warfarin. The surgery was performed promptly to avoid prolonged exposure to pus, which could have worsened to result in fibrosis. Surgery for tuboovarian abscess can be technically difficult while the necrotic tissue is difficult to handle as the fragile resulting in the tissue collapsing and hemorrhaging. The peritoneum is also often edema of the tissue, which results in challenging visualization of essential structures like the ureter. Moreover, the bowel is generally found to be adherent to structures in the pelvis when there is a tuboovarian abscess patient, consequently increasing the risk of visceral injury (3). Therefore, hydrodissection is a safer alternative, involves the infiltration of sterile water under low pressure between the parietal peritoneum and underlying retroperitoneal structures providing a safe and natural plane for dissection. In this case, the hydrodissection was surgery at the extensive adhesion of the obliteration of the pouch of Douglas without any complications. As such, laparoscopy is suitable for a complicated, high-risk patient, as laparoscopy can cause less bleeding, provide a quicker recovery, incur a smaller incision, less infection rate, and have reduced post-operative pain when compared to laparotomy. Nonetheless, the post-operative discharge was on Day 7 owing to the bridging therapy of the anticoagulant medication.

\section{Conclusions}

Laparoscopy is an alternatively safe for an extensive adhesion patient with a right tuboovarian abscess and a left mature teratoma by an experienced endoscopist which can provide a better security control for a complicated, high-risk patient.
Henceforth, laparoscopy can cause less bleeding, provide a quicker recovery, incur a smaller incision, less infection rate, and have reduced post-operative pain when compared with laparotomy.

*: Acknowledgements: The authors appreciate the AsiaPacific Association for Gynaecologic Endoscopy and Minimally Invasive Therapy (APAGE) for providing the International Fellowship Endoscopy Training Program at Chang Gung Memorial Hospital for Dr. Phornsawan Wasinghon.

Disclosure Statement: The authors declare that there are no conflicts of interest related to the current work.

\section{References}

1. Rosen M, Breitkopf D, Waud K. Tubo-ovarian abscess management options for women who desire fertility. Obstet Gynecol Surv 2009;64(10):681-9. doi: 10.1097/ OGX.0b013e3181b8b0d6.

2. Landers DV, Sweet RL. Tuboovarian abscess: contemporary approach to management. Rev Infect Dis 1983;5(5): 876-84

3. Munro K, Gharaibeh A, Nagabushanam S, Martin C. Diagnosis and management of tuboovarian abscess. The Obstetrician \& Gynaecologist 2018;20(1):11-19.

4. Chappell CA, Wiesenfeld HC. Pathogenesis, diagnosis, and management of severe pelvic inflammatory disease and tuboovarian abscess. Clin Obstet Gynecol 2012;55(4): 893-903.

5. Timor-Tritsch IE, Lerner JP, Monteagudo A, Murphy KE, Heller DS. Transvaginal sonographic markers of tubal inflammatory disease. Ultrasound Obstet Gynecol 1998; 12(1):56-66.

6. Crespo FA, Ganesh D, Lo K, Chin K, Norris P, Chakhtoura N. Surgical, ultrasound guided drainage, and medical management of tuboovarian abscesses. ISRN Infect Dis. 2014 March [cited 2018 September 1]. Available from: http://dx.doi.org/10.1155/2014/501729

7. Huang A, Jay MS, Uhler M. Tuboovarian abscess in the adolescent. J Pediatr Adolesc Gynecol 1997;10(2):73-7.

8. Mos C. Ovarian dermoid cysts: ultrasonographic findings. Medical Ultrasonography 2009;11(4):61-6.

9. Granberg S, Gjelland K, Ekerhovd E. The management of pelvic mass. Best Pract Res Clin Obstet Gynaecol 2009;23(5):667-678.

10. Nishimura RA, Otto TM, Bonow RO, Carabello BA, Erwin III JP, Fleisher LA, et al. Clinical practice guideline: focus update 2017 AHA/ACC focused update of the 2014 AHA/ACC guideline for the management of patients with valvular heart disease. J Am Coll Cardiol 2017; 70(2):252-89. 